\title{
DYNAMICAL SYSTEMS WITH A CONTINUUM OF RANDOMLY MATCHED GAMES*
}

\section{Carlos Alós-Ferrer**}

WP-AD 98-08

Correspondence to:

Carlos Alós-Ferrer. Department of Economics.University of Vienna.Hohenstaufengasse, 9. 1010 Vienna (Austria)

Editor: Instituto Valenciano de Investigaciones Económicas, S.A.

Primera Edición Abril 1998

ISBN: $84-482-1770-5$

Depósito Legal: V-1167-1998

IVIE working-papers offer in advance the results of economic research under way in order to encourage a discussion process before sending them to scientific journals for their final publication.

* I gratefully acknowledge S. Chattopadhyay, F. Marhuenda, J. Naeve, L. Samuelson, K.R. Schenk-Hoppé and F. Vega-Redondo for helpful comments.

** University of Alicante. 


\title{
DYNAMICS SYSTEMS WITH A CONTINUUM OF RANDOMLY MATCHED AGENTS
}

\author{
Carlos Alós-Ferrer
}

\begin{abstract}
Many models postulate a continuum of agents of finitely many different types who are repeatedly randomly matched in pairs to perform certain activities (e.g. play a game) which may in turn make their types change. The random matching process is usually left unspecified, and some Law of Large Numbers is informally invoked to justify a deterministic approximation of the resulting stochastic system. Nevertheless, it is well-known that such "laws of large numbers" may not hold in this framework. This work shows that there exist random matching processes over a continuum of agents satisfying properties which are sufficient to simplify the analysis of the stochastic system. Moreover, the evolution of the population frequencies of types induced by this system can be described (almost surely) through a set of deterministic equations.
\end{abstract}

Key words: Evolution; Random Matching; Law of Large Numbers; Dynamical Systems. 


\section{INTRODUCTION}

Many models both in economics and in biology study large populations where individuals of different types are randomly matched in pairs. In particular, in Evolutionary Game Theory, it is customary to postulate a "large" population of agents, programmed ("hardwired") to play certain pure strategies in a given finite, symmetric game (see e.g. Maynard Smith (1982) or Weibull (1995)). Types are then identified with strategies and, thus, there is a finite total number of types. The aim is precisely to study the evolution of the frequencies of these types in the population.

In this framework, individuals are repeatedly randomly matched in pairs to play the underlying finite game, and the outcomes of the game determine the fitness of the different types or strategies and how their frequencies change after each actual matching. The precise relationship between the actual play of the game and the evolution of the frequencies of types in the population depends on the specific model considered. For example, the well-known Replicator Dynamics is obtained by postulating the change in population frequencies to be proportional to the differences between average payoffs for each type and the population average payoff.

The focus of our work is not any specific model, but rather the properties of the random matching schemes which are implicitly used in many population models. Random matching creates a very complicated stochastic system. But, assuming that the population is large enough to make individual uncertainty negligible and that the number of types is finite, one hopes to summarize the evolution of the system by a finite number of deterministic equations. In order to do so, the random matching process is usually left unspecified, and then it is claimed that, by virtue of some Law of Large Numbers, the system can be studied through a deterministic approximation where the frequencies of different matches are identified with their corresponding expectations.

More rigorously, it is assumed that the proportion of matches between agents of two given types is equal to (twice) the product of the proportions of agents of those types. To illustrate this assumption, consider the following example: assume a large population of individuals of two types, 1 and 2 , and denote by $x$ the frequency of individuals of type 1 . What is usually assumed is that the random matching scheme is such that the frequency of matches between two individuals of type 1 is $x^{2}$; between two individuals of type 2 is $(1-x)^{2}$; and between individuals of different types is $2 x(1-x)$.

Another key assumption is that all individuals face the same distribution over possible partners, that is, the random matching process exhibits some "uniformity". More concretely, it is assumed that the probability with which a given agent is matched with some agent of a given type equals the frequency of agents of that type in the population. For instance, if the agents are matched to play an underlying 
game, this is often crucially used to identify payoffs with expected payoffs.

Despite their widespread use, rigorously stating and justifying these assumptions entails severe difficulties. The aim of this paper is precisely to undertake the analysis of their feasibility and implications in the case of a continuum population.

Why a continuum? The first difficulty which appears in random matching models is what is the precise meaning of a "large enough" population. There are at least three possible answers to this question, which create three different approaches to population models: finite, countably infinite, or a continuum.

The first answer is "large, but finite". Obviously, there is no problem to construct random matching schemes for finite populations, but then no "Law of Large Numbers" applies. The focus is then to study the limit behavior of the system as the population grows to infinity. Boylan (1995) rigorously studies the implications of this approach. The main criticism to this procedure is that, typically, there is no limit measure space of agents on which to define a limit stochastic process.

Nevertheless, in the evolutionary literature, population is often explicitly assumed to be infinite from the very beginning, in an attempt to implicitly use some Law of Large Numbers to transform the individual uncertainty created by random matching into aggregate certainty. But very rarely is anything more specified about the cardinality of the population, although it seems clear that there could be qualitative differences between a countably infinite population and an uncountable one.

For countably infinite populations, Boylan (1992) shows that there does not exist any random matching process satisfying a list of apparently innocuous properties, among them independence of the process from the specific types of agents and tentative formulations of some Law of Large Numbers. However, Boylan also shows that it is possible to construct random matching processes that verify some minimal properties which are enough to justify the usual claims about random matching, analogous to the two assumptions discussed above. This construction relies on the use of Kolmogorov's Strong Law of Large Numbers, which applies because the population is countable, and depends on the specific assignment of types in the population. Moreover, Boylan proves that this dependence on the assignment of types is unavoidable when the population is countably infinite.

Gilboa and Matsui (1992) construct a model of random matching for countably infinite populations using finitely additive measures. The random matching scheme of this model does not depend on the assignment of types, and all individuals face the same distribution over possible partners. Nevertheless, the measure space fails to be fully sigma-additive, and sigma-additivity seems necessary to establish the properties guaranteed by Boylan (more concretely, the properties which would allow to identify the frequencies of different matches with their corresponding expectations).

Many random matching models, though, do not consider the population to be countable, but rather assume a continuum of individuals. Among them are Matsui 
and Matsuyama (1995) or Harrington (1997), as examples in the evolutionary literature, but also some models in other branches of economics, e.g. Peters $(1991,1997)$ and McLennan and Sonnenschein (1991) in continuum economies. ${ }^{1}$

The advantages of a continuum population are twofold. On the one hand, it could be argued that it provides the best idealization for situations where individuals are thought to be insignificant with respect to the population (e.g., if the individuals perceive the situation as if the population were infinite), or uniform distributions are called for. One of the first problems of random matching with a countably infinite population is that individuals cannot face uniform marginal distributions over partners, i.e. not all the other individuals can be equiprobable as partners. This is due to the inexistence of uniform distributions over a countably infinite number of events. With a continuum of individuals, this difficulty does not exist, and it will be shown that properties which can never be satisfied with a countable population hold in the case of a continuum.

On the other hand, there is the issue of analytical tractability. The technical tools needed to study a continuum population are those of measure theory, which are firmly established in the economic literature. E.g., the frequency of individuals of a given type in a continuum population is just its Lebesgue measure, while in a countably infinite one, it would call for the use of Cesaro averages, i.e. limits of averages over finite fractions of the population.

In any case, it could be argued that the importance of the continuum idealization for economic theory is nowadays sufficiently justified by the existence of models that explicitly make use of random matching schemes for continuum populations, as those cited before. Nevertheless, rigorous analysis of its implications has not yet been undertaken.

Unfortunately, this analysis has been more or less implicitly considered hopeless, due to the well-known problems that arise with a continuum of random variables, as illustrated by Judd (1985). In fact, Feldman and Gilles (1985) proved that there is simply no Law of Large Numbers for a continuum of random variables. In particular, with a continuum population, Boylan's construction would fail where it invokes Kolmogorov's Law.

Despite these difficulties, this paper proves that it is possible to construct random matching processes for a continuum of agents satisfying a number of properties which suffice to justify the claims of many random matching models, even though no general Law of Large Numbers holds. Those models can then be justified, although not by the usually mentioned arguments (i.e. "Law of Large Numbers").

\footnotetext{
${ }^{1}$ Some of these models, however, depart from the basic random matching assumptions. The models by Peters, e.g., assume random matching between a population of sellers and a population of buyers of different measures.
} 
It is also shown that, analogously to Boylan's results, such random matching processes have necessarily to depend on the specific assignment of types in the population.

The last part of the paper characterizes the evolution of the population frequencies of types in dynamical systems with repeated random matching, and shows that these systems can be described through a finite set of deterministic equations, which is precisely one of the basic implicit assumptions in Evolutionary Game Theory.

\section{NOTATION}

We consider a continuum of agents. Without loss of generality, let us take the halfopen interval $(0,1]$ to be the set of agents. ${ }^{2}$ Let also $\mathbb{B}$ be the Borel $\sigma$-algebra on $(0,1]$, and $\mu$ the Lebesgue measure. Then, the space of agents will be the measure space $((0,1], \mathbb{B}, \mu)$.

Let $S=\left\{s_{1}, \ldots, s_{m}\right\}, m \geq 2$, be the set of possible types of agents, assumed to be finite. For notational convenience, and following the notation of Boylan (1992), identify $S$ with the canonical basis of $\mathbb{R}^{m}$, i.e., $s_{r}=(0, \ldots, 0, \stackrel{(r)}{1}, 0, \ldots 0)^{\prime}$.

An assignment of types is a function $\alpha:(0,1] \rightarrow S$, mapping each agent $i$ to its type $\alpha(i)$, such that the sets of agents of a given type are measurable and have positive measure, i.e., $I_{r}(\alpha)=\alpha^{-1}\left(s_{r}\right)=\left\{i \in(0,1] / \alpha(i)=s_{r}\right\} \in \mathbb{B}$ and $P_{r}=$ $\mu\left(I_{r}(\alpha)\right)>0 \forall r=1, \ldots, m$.

Denote by $\alpha_{r}(i)$ the $r$-th coordinate of the vector $\alpha(i)$, so that $\alpha_{r}(i)=1$ if and only if agent $i$ is of type $s_{r}$.

Construct a probability measure on $S$ by $\lambda_{\alpha}(T)=\sum_{s_{r} \in T} P_{r}$. Obviously, $\left(S, 2^{S}, \lambda_{\alpha}\right)$ is a probability space such that $\alpha$ is a measurable function.

We are interested in constructing random matching schemes, i.e. probability spaces over matchings. A matching is a way to assign to each agent in the population a partner in such a way that all agents have a partner, the partner of the partner of an agent is the agent himself, and no agent is his own partner. In summary:

Definition 1. A matching is any element of the set

$$
\sum=\left\{\sigma:(0,1] \rightarrow(0,1] \text { bijective } / \forall i \in(0,1], \sigma^{2}(i)=i \neq \sigma(i)\right\}
$$

Definition 2. A random matching scheme is a probability space $\left(\sum, \mathcal{F}, P\right)$ where $\mathcal{F}$ is a $\sigma$-algebra on $\sum$ and $P$ is a countably additive probability measure.

\footnotetext{
${ }^{2}$ The set of agents is taken to be the half-open unit interval for notational and interpretative convenience (is easy to think of this interval as a circumference), but the analysis could be easily adapted to the closed or open interval. Also, Lebesgue measure is used for the sake of clarity, but any nonatomic measure could be used instead.
} 
The following is a list of properties which are usually assumed in the literature, either explicitly or implicitly. Note that several of them depend on $\alpha$.

- "Minimal" Properties:

P1 (Measure Preservation): $\mu(\sigma(E))=\mu(E) \forall E \in \mathbb{B}, P$-almost surely.

P2 (Types' Proportional Law): $\forall i \in(0,1], \forall s_{r} \in S, P\left(\alpha(\sigma(i))=s_{r}\right)=P_{r}$.

P3 (Types' Law of Large Numbers): $\forall s_{r}, s_{v} \in S, \int_{(0,1]} \alpha_{r}(i) \alpha_{v}(\sigma(i)) d \mu=P_{r} P_{v} P_{-}$ almost surely.

- "General" Properties:

P4 (General Proportional Law): $\forall i \in(0,1], \forall E \in \mathbb{B}, P(\sigma(i) \in E)=\mu(E)$.

P5 (Strong Law of Large Numbers): $\mu\left(E_{1} \cap \sigma\left(E_{2}\right)\right)=\mu\left(E_{1}\right) \mu\left(E_{2}\right) \forall E_{1}, E_{2} \in \mathbb{B}$ $P$-almost surely.

The first three properties are the minimal requirements that a random matching scheme should verify, in order for the usual claims in the literature to hold. The first one simply states that the measure of a given Borel set of agents should coincide (almost surely) with the measure of the set of agents who are matched with them. More concretely, P1 says that the measure of the matchings that are measure-preserving, i.e. that verify this property for all Borel sets, is equal to one. This is necessary because, otherwise, it would be possible to construct matchings as bijections between sets of different measures, e.g. to match all the agents in the interval $\left(0, \frac{1}{10}\right]$ with all the agents in $\left(\frac{1}{10}, 1\right]$.

The second means that the probability with which a given agent is matched with some agent of a specific type should coincide with the measure of agents of that type in the population. This property is generally assumed to hold as a consequence of some uniformity in the matching process, and is necessary e.g. to link the frequencies of types to the expected payoffs of a given agent.

The third property states that the proportion of matches between agents of two specific types is (almost surely) the product of the proportions of agents of both types (a " 2 " is missing because the expression is asymmetric in types). This is the key property to write down the deterministic equations which (hopefully) summarize the evolution of population frequencies in a given model, once this model specifies how each possible match affects the types of the involved individuals (or its descendants, in a more evolutionary interpretation). 
P4 is often informally stated by saying that all agents face the same marginal distribution over possible partners, and these distributions are uniform. It is the formalization of the sentence "agents are randomly uniformly matched".

P5 is taken from McLennan \& Sonnenschein (1991, p. 1409) as the tentative formulation of a Law of Large Numbers for this framework, and states that almost all the matchings have the following property: the measure of the agents in a given Borel set who are matched with agents in another Borel set is the product of the measures of the two sets (see Appendix 2 for some additional discussion on this property).

Obviously, P4 implies P2 and P5 implies P3. Sometimes, P4 and P5 are seen as justifications for P2 and P3, and are assumed to be in turn justified by some Law of Large Numbers. In fact, the key properties for many random matching models are just $\mathrm{P} 2$ and $\mathrm{P} 3$.

\section{Negative Results}

The following proposition proves that there is no hope of constructing a random matching scheme satisfying the more general Law of Large Numbers, so attention should be restricted to the "minimal" properties. In particular, it shows that, given $\mathrm{P} 1$, then P5 is impossible to be fulfilled. This proposition is taken and adapted from McLennan and Sonnenschein (1991), and the proof is included only for completeness: although it is possible to provide other proofs of the same fact, this one has the virtue of linking the impossibility result directly to the problems illustrated by Feldman \& Gilles (1985).

Proposition 1. (McLennan \& Sonnenschein, 1991) There exists no random matching scheme satisfying $P 1$ and $P 5$.

Proof. Suppose $\left(\sum, \mathcal{F}, P\right)$ is a random matching scheme satisfying $\mathrm{P} 1$ and P5. By P5, we can choose a matching $\sigma \in \sum$ such that $\forall E_{1}, E_{2} \in \mathbb{B}, \mu\left(E_{1} \cap\right.$ $\left.\sigma\left(E_{2}\right)\right)=\mu\left(E_{1}\right) \mu\left(E_{2}\right)$. By P1, we can assume that this $\sigma$ is such that $\mu\left(\sigma\left(\left(0, \frac{1}{2}\right]\right)\right)=$ $\mu\left(\left(0, \frac{1}{2}\right]\right)=\frac{1}{2}$.

Let $\chi_{\left(0, \frac{1}{2}\right]}$ be the characteristic function of the set $\left(0, \frac{1}{2}\right]$, and define $\left.X=\chi_{\left(0, \frac{1}{2}\right]}\right]^{\circ \sigma}$. Then, $\forall E \in \mathbb{B}$,

$$
\begin{aligned}
\mu\left(E \cap X^{-1}(1)\right) & =\mu\left(E \cap \sigma^{-1}\left(\left(0, \frac{1}{2}\right]\right)\right)=\mu\left(E \cap \sigma\left(\left(0, \frac{1}{2}\right]\right)\right)= \\
& =\mu(E) \mu\left(\sigma\left(\left(0, \frac{1}{2}\right]\right)\right)=\frac{1}{2} \mu(E)
\end{aligned}
$$

First equality: definition of $X$; second: $\sigma^{2}$ is the identity; third: P5; fourth: P1. 
But such a function $X$ cannot exist by Proposition 1 in Feldman and Gilles (1985)

This proposition shows, not only that $\mathrm{P} 1$ and $\mathrm{P} 5$ are incompatible, but also that there does not exist any matching satisfying simultaneously the properties described in $\mathrm{P} 1$ and $\mathrm{P} 5$. Clearly, there is no hope of constructing random matching schemes satisfying $\mathrm{P} 5$.

One further useful property would be that the random matching scheme does not depend on the agents' types, that is, that the same random matching scheme could be used for different assignments of types and still satisfy some of those minimal properties the statement of which depends on the agents' types, e.g. P2 and P3. The following corollary shows that this is not possible. Concretely, if a random matching scheme satisfies P1 and P3, then, necessarily, it has to depend on the assignment of types, $\alpha$. This conclusion is similar to that of Boylan (1992) for countably infinite populations, although the reasons are different.

The main argument of the proof is simple. If we require a random matching scheme not do depend on the assignment of types, then satisfying P3 would mean that P3 should hold for any assignment of types. Then, given two arbitrary Borel subsets of the space of agents, we can construct an assignment of types such that the statement of P3 is equivalent to the statement of P5 for these two specific subsets. Since this is possible for any arbitrary pair of Borel subsets, we see that P3 and independence of the assignment of types would imply P5. This traces back the impossibility to the preceding proposition, and, thus, to the basic impossibility result of Feldman \& Gilles (1985).

Corollary 2. There exists no random matching scheme satisfying $P 1$ and P3 which does not depend on the assignment of types (i.e., which satisfies P3 for all possible $\alpha)$.

Proof. Suppose first that there are at least three types. Assume, for the sake of contradiction, that such a random matching scheme exists. Then, in view of the above proposition, it suffices to show that it will also satisfy P5.

First, we establish P5 $\forall E_{1}, E_{2} \in \mathbb{B} / E_{1} \cap E_{2}=\emptyset:$ Define $\alpha:(0,1] \rightarrow S$ such that $\alpha(i)=s_{1} \forall i \in E_{1}, \alpha(i)=s_{2} \forall i \in E_{2}, \alpha(i)=s_{3} \forall i \notin E_{1} \cup E_{2}$. Let $\chi_{A}$ be the characteristic function of a set $A$. Then, for almost all $\sigma$,

$$
\begin{aligned}
\mu\left(E_{1} \cap \sigma\left(E_{2}\right)\right) & =\int_{(0,1]} \chi_{E_{1}}(i) \chi_{\sigma\left(E_{2}\right)}(i) d \mu= \\
& =\int_{(0,1]} \alpha_{1}(i) \alpha_{2}(\sigma(i)) d \mu=P_{1} P_{2}=\mu\left(E_{1}\right) \mu\left(E_{2}\right)
\end{aligned}
$$


Second, let $E_{1}, E_{2} \in \mathbb{B} / E_{1} \cap E_{2} \neq \emptyset$. Decompose $E_{1}=\left(E_{1} \cap E_{2}\right) \cup\left(E_{1} \backslash E_{2}\right)$. Then,

$$
\begin{aligned}
\mu\left(E_{1} \cap \sigma\left(E_{2}\right)\right) & =\mu\left(\left[\left(E_{1} \cap E_{2}\right) \cap \sigma\left(E_{2}\right)\right] \cup\left[\left(E_{1} \backslash E_{2}\right) \cap \sigma\left(E_{2}\right)\right]\right)= \\
& =\mu\left(E_{2}\right)\left[\mu\left(E_{1} \cap E_{2}\right)+\mu\left(E_{1} \backslash E_{2}\right)\right]=\mu\left(E_{1}\right) \mu\left(E_{2}\right)
\end{aligned}
$$

which completes the proof when there are at least three different types.

If there are only two types, it would suffice to show that, if a random matching scheme satisfying P1 and P3 for all possible assignments of two types $\alpha$ would exist, then the same random matching scheme would also satisfy P3 for all possible assignments of three types $\beta$.

More concretely, let $\left(\sum, \mathcal{F}, P\right)$ be a random matching scheme satisfying $\mathrm{P} 1$ and $\mathrm{P} 3$ for all possible assignments of types $\alpha$ with two types. Now, let $\beta$ be an assignment of types with three types, $s_{1}, s_{2}$, and $s_{3}$, and let $E_{r}=I_{r}(\beta)$. Let, for a given $\sigma$,

$$
X_{r v}=\mu\left(E_{r} \cap \sigma\left(E_{v}\right)\right)-P_{r} P_{v} \quad \forall r, v=1,2,3
$$

The proof would be complete if it is shown that $X_{r v}=0 P$-almost surely $\forall r, v=$ 1, 2,3. First of all, note that, by P1,

$$
\begin{aligned}
X_{r v} & =\mu\left(E_{r} \cap \sigma\left(E_{v}\right)\right)-P_{r} P_{v}=\mu\left(\sigma\left(E_{r} \cap \sigma\left(E_{v}\right)\right)\right)-P_{v} P_{r}= \\
& =\mu\left(E_{v} \cap \sigma\left(E_{r}\right)\right)-P_{v} P_{r}=X_{v r}
\end{aligned}
$$

with probability one. It's also easy to see that, by P3,

$$
\mu\left(E_{r} \cap \sigma\left(E_{r}\right)\right)=P_{r}^{2}
$$

and thus $X_{r r}=0 \forall r$. In summary,

$$
\begin{aligned}
& X_{11}=X_{22}=X_{33}=0 \\
& X_{12}=X_{21} ; X_{13}=X_{31} ; X_{23}=X_{32}
\end{aligned}
$$

so, all that remains to be shown is that $X_{12}=X_{13}=X_{23}=0$.

But notice that, given the three types $r, v, w$, it is possible to define a new assignment of types $\alpha_{v w}^{\prime}$ with only two types such that $\alpha_{v w}^{\prime}(i)=s_{r}$ iff $\beta(i)=s_{r}$ and $\alpha_{v w}^{\prime}(i)=s_{v}$ otherwise. Then, P3 holds for $\alpha_{v w}^{\prime}$ with probability one, and thus, given that the sets $E_{r}, E_{v}, E_{w}$ are disjoint and $\sigma$ is bijective,

$$
\mu\left(E_{r} \cap \sigma\left(E_{v}\right)\right)+\mu\left(E_{r} \cap \sigma\left(E_{w}\right)\right)=\mu\left(E_{r} \cap \sigma\left(E_{v} \cup E_{w}\right)\right)=P_{r}\left(P_{v}+P_{w}\right)
$$

which implies that, with probability one,

$$
X_{r v}+X_{r w}=0 \quad \forall r, v=1,2,3, r \neq v
$$


giving us three equations which immediately imply $X_{12}=X_{13}=X_{23}=0$, the desired conclusion.

The last corollary proves that, in order to construct random matching schemes satisfying at least P1, P2, and P3, it is necessary to allow them to depend on the assignment of types. This result is similar to one of Boylan (1992) for countably infinite populations.

\section{Positive Results}

Boylan constructs a random matching scheme for countably infinite populations which depends on the assignment of types. This dependence can be summarized by the existence of a "weighted die", with types on its faces, such that the probability of each type is numerically equal to the proportion of agents of that type in the population, as given by the assignment of types.

Boylan's construction works as follows. Agents are assigned natural numbers and then, they throw the same weighted die sequentially. This procedure can be thought of as a sequence of i.i.d. discrete random variables, which verifies Kolmogorov's Strong Law of Large Numbers (see e.g. [1, Theorem 7.2.2]).

For each agent, the type he rolls is recorded. If a given agent of type $s_{r}$ has rolled an $s_{v}$ and there exists an agent with an strictly lower label, which is of type $s_{v}$ and has rolled an $s_{r}$, then these two agents are matched. If it does not exist such a second agent, then the first waits to be matched.

This procedure depends crucially on the fact that the population is countable. Also, all the properties of the random matching schemes are derived from the fact that Kolmogorov's Strong Law of Large Numbers applies to the sequence of dice rolls. Obviously, there is no hope of applying a similar procedure to a continuum population. Nevertheless, as it is shown below, it is possible to use a totally different approach which does not rely on any Law of Large Numbers.

The following technical lemma is crucially used in the proof of the main theorem.

Lemma 3. Let $E_{1}, E_{2} \in \mathbb{B} / \mu\left(E_{1}\right)=\mu\left(E_{2}\right)>0$. Then, $\exists \varphi: E_{1} \rightarrow E_{2}$ bijective and measurable with measurable inverse and such that $\forall A \in \mathbb{B} \cap E_{2}, \mu\left(\varphi^{-1}(A)\right)=$ $\mu(A) . \varphi$ is called a measure-preserving bijection ${ }^{3}$.

\section{Proof. See Appendix 1.}

This lemma allows us to work "as if" the agents could be rearranged. E.g., any Borel set of measure $K>0$ could be fictitiously transformed in the half-open interval

\footnotetext{
${ }^{3}$ As customary in measure theory, $\mathbb{B} \cap E_{2}$ denotes the trace $\sigma$-algebra.
} 
$(0, K]$ for our purposes, since this transformation can be done through measurepreserving bijections. Moreover, the proof of the lemma is constructive, giving us "canonical" transformations.

The idea of the random matching construction which proves the next theorem is simple. The dependence of the random matching scheme from the assignment of types is going to be summarized in the existence of a device called "marked circumference", which is somewhat analogous to Boylan's weighted die. This can be thought of as a circumference of perimeter 1 , such that, for each type $s_{r}$, there exists a half-open arc on the circumference, exactly of length $P_{r}$, which is labeled $s_{r}$.

The procedure works as follows. First, rearrange the agents in the population through Lemma 3 so that all agents of the same type are in a half-open interval of the appropriate measure (of course, this is not really done, but Lemma 3 allows us to think of the procedure as if it was). Each of these intervals can be considered as a circumference. So, we have $m$ circumferences, one for each type of agents, and the circumference for agents of type $s_{r}$ is of perimeter $P_{r}$.

Second, homothetically "expand" these circumferences until their perimeters are all 1, and then randomly roll them (is tempting to think of the procedure as "the roulette method"). Place each one of these circumferences over the marked circumference. Each agent, after expanding the circumference of his type and rolling it, is placed over some of the labels of the marked circumference. Record the corresponding label for each agent.

Third, consider the set of agents of type $s_{r}$ who have been placed over locations labeled $s_{v}$ on the marked circumference after their own circumference has been rolled. Conversely, consider the set of agents of type $s_{v}$ who have rolled an $s_{r}$. By construction, it is easy to see that both sets have the same (positive) measure, so agents in them can be matched through a measure-preserving bijection by virtue of Lemma 3 .

Theorem 4. For any assignment of types $\alpha$, there exists a random matching scheme satisfying $P 1, P 2$, and $P 3$.

Proof. Denote the elements of $(0,1]$ by $\omega_{r}$ (the subindex is to avoid confusion with vectors, denoted by $\omega)$. Let $Z:(0,1] \rightarrow S$ be such that $Z\left(\omega_{r}\right)=s_{v} \Leftrightarrow$ $\omega_{r} \in\left(\sum_{j=1}^{v-1} P_{j}, \sum_{j=1}^{v} P_{j}\right]$. I.e., $Z^{-1}\left(s_{v}\right)=\left(\sum_{j=1}^{v-1} P_{j}, \sum_{j=1}^{v} P_{j}\right]$. Obviously, $Z$ is measurable.

The key property of $Z$ is that $\mu \circ Z^{-1}=\lambda_{\alpha}$ (in fact, the proof remains valid for any measurable function satisfying this property).

For each $s_{r} \in S$, define a family of $S$-valued random variables $\left\{X_{i} / i \in I_{r}(\alpha)\right\}$ by

$$
X_{i}:(0,1] \rightarrow S / X_{i}\left(\omega_{r}\right)= \begin{cases}Z\left(\varphi_{r}(i)+\omega_{r}\right) & \text { if } \varphi_{r}(i)+\omega_{r} \leq 1 \\ Z\left(\varphi_{r}(i)+\omega_{r}-1\right) & \text { if } \varphi_{r}(i)+\omega_{r}>1\end{cases}
$$


where $\varphi_{r}=\varphi_{r}^{2} \circ \varphi_{r}^{1}$, with $\varphi_{r}^{1}: I_{r}(\alpha) \rightarrow\left(0, P_{r}\right]$ a measure-preserving bijection (which exists by Lemma 3$)$ and $\varphi_{r}^{2}:\left(0, P_{r}\right] \rightarrow(0,1]$ such that $\varphi_{r}^{2}(x)=\frac{1}{P_{r}} x \forall x$.

Construct now the product measure space $\left((0,1]^{m}, \mathbb{B}^{m}, \mu^{m}\right) \stackrel{P_{r}}{=} \prod_{j=1}^{m}((0,1], \mathbb{B}, \mu)$, which represents the realization of $m$ independent random variables with values in $(0,1]$ and uniform distributions.

Given $\omega \in(0,1]^{m}$, let

$$
X(\omega)=\left\{\left\{X_{i}\left(\omega_{r}\right)\right\}_{i \in I_{r}(\alpha)}\right\}_{r=1}^{m}
$$

Notice that $X$ is injective as a function from $(0,1]^{m}$ to $S^{(0,1]} .^{4}$

Our aim is to construct a matching such that each agent $i$ of type $s_{r}$ is matched with some agent of type $X_{i}\left(\omega_{r}\right)$. In fact, we could construct now the probability space $\left(S^{(0,1]}, \mathcal{M}, \nu\right)$, with $\mathcal{M}$ the $\sigma$-algebra of $S^{(0,1]}$ generated by $X\left(\mathbb{B}^{m}\right)$, and $\nu=\mu^{m} \circ X^{-1}$. This space represents the draw of a tentative assignment of agents to types, i.e., for each agent, the type of its future partner is drawn.

Define, $\forall s_{r}, s_{v} \in S, \forall \omega \in(0,1]^{m}$,

$$
A_{r v}(\alpha, \omega)=\left\{i \in(0,1] / \alpha(i)=s_{r}, X_{i}\left(\omega_{r}\right)=s_{v}\right\}
$$

So, with the above interpretation for $X_{i}\left(\omega_{r}\right)$, each set $A_{r v}(\alpha, \omega)$ can be interpreted as the set of agents of type $s_{r}$ who have to be matched with agents of type $s_{v}$.

Let us show some properties of the random variables $X_{i}\left(\omega_{r}\right)$ and the sets $A_{r v}(\alpha, \omega)$ :

1. $X_{i}$ is measurable $\forall i \in I_{r}(\alpha)$.

Let $k=\varphi_{r}(i)$. Define a function $C(k):(0,1] \rightarrow(0,1]$ such that

$$
C(k)\left(\omega_{r}\right)= \begin{cases}k+\omega_{r} & \text { if } k+\omega_{r} \leq 1 \\ k+\omega_{r}-1 & \text { if } k+\omega_{r}>1\end{cases}
$$

Obviously, $C(k)$ is measurable. Note that $X_{i}=Z \circ C(k)$. So, $X_{i}$ is a composition of measurable functions and hence measurable. ${ }^{5}$

\footnotetext{
${ }^{4}$ In fact, $\left\{X_{i}\left(\omega_{r}\right)\right\}_{i \in I_{r}(\alpha)}$ is injective. Let $0<\omega_{r}<\omega_{r}^{\prime} \leq 1$. If $\omega_{r}^{\prime}-\omega_{r} \leq 1-P_{1}$, then let $x=P_{1}-\omega_{r}$ if $P_{1}-\omega_{r}>0$, or $x=P_{1}-\omega_{r}+1$ if not. Then, $X_{\varphi^{-1}(x)}\left(\omega_{r}\right)=Z\left(P_{1}\right)=s_{1}$, and $X_{\varphi^{-1}(x)}\left(\omega_{r}^{\prime}\right)=Z\left(P_{1}+\left(\omega_{r}^{\prime}-\omega_{r}\right)\right) \neq s_{1}$, because $P_{1}<P_{1}+\left(\omega_{r}^{\prime}-\omega_{r}\right) \leq 1$.

If $\omega_{r}^{\prime}-\omega_{r}>1-P_{1}$, then let $x=1-\omega_{r}^{\prime}$. Then, $X_{\varphi^{-1}(x)}\left(\omega_{r}^{\prime}\right)=Z(1) \neq s_{1}$, and $X_{\varphi^{-1}(x)}\left(\omega_{r}\right)=$ $Z\left(1-\left(\omega_{r}^{\prime}-\omega_{r}\right)\right)=s_{1}$, because $0<1-\left(\omega_{r}^{\prime}-\omega_{r}\right)<P_{1}$.

${ }^{5} \mathrm{It}$ is not difficult to see that $X_{i}^{-1}\left(s_{v}\right)=\left(\max \left(0, \sum_{j=1}^{v-1} P_{j}-k\right), \sum_{j=1}^{v} P_{j}-k\right] \cup$ $\left(1+\sum_{j=1}^{v-1} P_{j}-k, \min \left(1,1+\sum_{j=1}^{v} P_{j}-k\right)\right]$
} 
2. $\forall T \subset S, \forall i \in I_{r}(\alpha), \operatorname{Pr}\left(X_{i} \in T\right)=\mu\left(\left\{\omega_{r} \in(0,1] / X_{i}\left(\omega_{r}\right) \in T\right\}\right)=\lambda_{\alpha}(T)$.

It is enough to show this property for the elements of $S$, because this set is finite. Let $k=\varphi_{r}(i)$. Note that the function $C(k)$ as defined above is measurepreserving, i.e. $\mu\left(C(k)^{-1}(E)\right)=\mu(E) \forall E \in \mathbb{B}$.

Given $s_{v} \in S$,

$$
\begin{aligned}
\mu\left(X_{i}^{-1}\left(s_{v}\right)\right) & =\mu\left((Z \circ C(k))^{-1}\left(s_{v}\right)\right)=\mu\left(C(k)^{-1}\left(Z^{-1}\left(s_{v}\right)\right)\right)= \\
& =\mu\left(Z^{-1}\left(s_{v}\right)\right)=\left(\mu \circ Z^{-1}\right)\left(s_{v}\right)=\lambda_{\alpha}\left(s_{v}\right)=P_{v}
\end{aligned}
$$

3. $\forall T \subset S, \forall \omega_{r} \in(0,1], \mu\left(\left\{i \in I_{r}(\alpha) / X_{i}\left(\omega_{r}\right) \in T\right\}\right)=P_{r} \lambda_{\alpha}(T)$.

As before, it is enough to prove the claim for the elements of $S$. Given $s_{r} \in S$, and any $\omega \in(0,1]^{m}$ such that its $r$-th coordinate is $\omega_{r}$, we have that

$$
\begin{aligned}
\varphi_{r}\left(A_{r v}(\alpha, \omega)\right) & =\varphi_{r}\left(\left\{i \in I_{r}(\alpha) / X_{i}\left(\omega_{r}\right)=s_{v}\right\}\right)= \\
& =\left\{k \in(0,1] /(Z \circ C(k))\left(\omega_{r}\right)=s_{v}\right\}= \\
& =\left\{k \in(0,1] / C(k)\left(\omega_{r}\right) \in Z^{-1}\left(s_{v}\right)\right\}
\end{aligned}
$$

Notice that, by construction, $C(k)\left(\omega_{r}\right)=C\left(\omega_{r}\right)(k)$. Thus,

$$
\begin{aligned}
\mu\left(\varphi_{r}\left(A_{r v}(\alpha, \omega)\right)\right) & =\mu\left(\left\{k \in(0,1] / C\left(\omega_{r}\right)(k) \in Z^{-1}\left(s_{v}\right)\right\}\right)= \\
& =\mu\left(C\left(\omega_{r}\right)^{-1}\left(Z^{-1}\left(s_{v}\right)\right)\right)=\mu\left(Z^{-1}\left(s_{v}\right)\right)= \\
& =\left(\mu \circ Z^{-1}\right)\left(s_{v}\right)=\lambda_{\alpha}\left(s_{v}\right)=P_{v}
\end{aligned}
$$

and, by definition of $\varphi_{r}, A_{r v}(\alpha, \omega)$ has measure $P_{r} P_{v}$ and the conclusion follows.

We have already shown that the sets $A_{r v}(\alpha, \omega)$ inherit the properties of the variables $X_{i}\left(\omega_{r}\right)$ :

1. $A_{r v}(\alpha, \omega)$ is measurable.

2. $\forall i \in I_{r}(\alpha), \operatorname{Pr}\left(i \in A_{r v}(\alpha, \omega)\right)=\mu^{m}\left(\left\{\omega \in(0,1]^{m} / X_{i}\left(\omega_{r}\right)=s_{v}\right\}\right)=$ $=\mu\left(\left\{\omega_{r} \in(0,1] / X_{i}\left(\omega_{r}\right)=s_{v}\right\}\right)=\lambda_{\alpha}\left(\left\{s_{v}\right\}\right)=P_{v}$.

3. $\forall \omega \in(0,1]^{m}, \mu\left(A_{r v}(\alpha, \omega)\right)=P_{r} P_{v}$.

Let us construct now the matching that corresponds to a given $\omega \in(0,1]^{m}$.

Given $\omega \in(0,1]^{m}$, let $\varphi_{r v}: A_{r v}(\alpha, \omega) \rightarrow A_{v r}(\alpha, \omega)$ be a measure-preserving bijection $\forall s_{r} \neq s_{v}$, with $\varphi_{v r}^{-1}=\varphi_{r v}$, and let $\varphi_{r r}: A_{r r}(\alpha, \omega) \rightarrow\left(0, P_{r}^{2}\right]$ a measurepreserving bijection $\forall s_{r}\left(\varphi_{r v}, \varphi_{r r}\right.$ exist by Lemma 3 , which can be applied because 
of the properties just proven). Let also $\psi_{r}:\left(0, P_{r}^{2}\right] \rightarrow\left(0, P_{r}^{2}\right]$ be such that $\psi_{r}(x)=$ $x+\frac{1}{2} P_{r}^{2} \forall x \in\left(0, \frac{1}{2} P_{r}^{2}\right], \psi_{r}(x)=x-\frac{1}{2} P_{r}^{2} \forall x \in\left(\frac{1}{2} P_{r}^{2}, P_{r}^{2}\right]$.

Let $\sigma_{\alpha}^{\omega}:(0,1] \rightarrow(0,1] / \sigma_{\alpha}^{\omega}(i)= \begin{cases}\varphi_{r v}(i) & \text { if } i \in A_{r v}(\alpha, \omega), s_{r} \neq s_{v} \\ \left(\varphi_{r r}^{-1} \circ \psi_{r} \circ \varphi_{r r}\right)(i) & \text { if } i \in A_{r r}(\alpha, \omega)\end{cases}$

Obviously, $\sigma_{\alpha}^{\omega}$ is a matching, i.e. $\sigma_{\alpha}^{\omega} \in \sum$. We will also occasionally denote $\sigma_{\alpha}^{\omega}=$ $\sigma(\alpha, \omega)$ for convenience.

This procedure defines a mapping $\sigma_{\alpha}:(0,1]^{m} \rightarrow \sum$ which turns out to be injective: If $\omega, \omega^{\prime} \in(0,1]^{m} / \sigma_{\alpha}^{\omega}=\sigma_{\alpha}^{\omega^{\prime}} \Rightarrow X(\omega)=\alpha\left(\sigma_{\alpha}^{\omega}\right)=\alpha\left(\sigma_{\alpha}^{\omega^{\prime}}\right)=X\left(\omega^{\prime}\right) \Rightarrow \omega=\omega^{\prime}$ the last implication holds because $X$ itself is injective).

Let $\sum_{\alpha}=\sigma_{\alpha}\left((0,1]^{m}\right)$, i.e., identify the realizations of the $m$ i.i.d. random variables (and, thus, the tentative assignments of individuals to types) with the described matchings.

Let $\mathcal{F}_{\alpha}$ be the $\sigma$-algebra on $\sum_{\alpha}$ generated by $\sigma_{\alpha}\left(\mathbb{B}^{m}\right)$, and $P_{\alpha}=\mu^{m} \circ \sigma_{\alpha}^{-1}$.

Obviously, $\left(\sum_{\alpha}, \mathcal{F}_{\alpha}, P_{\alpha}\right)$ is a probability space. All what is left to do is to extend this to a probability space over the full set of matchings.

Let $\mathcal{F}$ be the $\sigma$-algebra generated by $\mathcal{F}_{\alpha}$ and $\sum \backslash \sum_{\alpha}$. For all $A \in \mathcal{F}$, define $P(A)=P_{\alpha}\left(A \cap \sum_{\alpha}\right)$

Then, $\left(\sum, \mathcal{F}, P\right)$ is a probability space over the full set of matchings and thus a random matching scheme.

Now we need to verify the claimed properties.

(P1) Is immediately satisfied thanks to the fact that all matchings in $\sum_{\alpha}$ are constructed via measure-preserving bijections.

(P2) Given $i \in(0,1]$, let $r$ be such that $i \in I_{r}(\alpha)$. Then,

$$
\begin{aligned}
P\left(\left\{\sigma \in \sum / \alpha(\sigma(i))=s_{v}\right\}\right) & =P_{\alpha}\left(\left\{\sigma \in \sum_{\alpha} / \alpha(\sigma(i))=s_{v}\right\}\right)= \\
\mu^{m}\left(\left\{\omega \in(0,1]^{m} / \alpha\left(\sigma_{\alpha}^{\omega}(i)\right)=s_{v}\right\}\right) & =\mu^{m}\left(\left\{\omega \in(0,1]^{m} / i \in A_{r v}(\alpha, \omega)\right\}\right)= \\
\mu^{m}\left(\left\{\omega \in(0,1]^{m} / X_{i}\left(\omega_{r}\right)=s_{v}\right\}\right) & =P_{v}
\end{aligned}
$$

so the random matching scheme satisfies the Proportional Law for types.

(P3) Given $\sigma=\sigma_{\alpha}^{\omega} \in \sum_{\alpha}$, and given $s_{r}, s_{v} \in S$,

$$
\begin{aligned}
\int_{(0,1]} \alpha_{r}(i) \alpha_{v}(\sigma(i)) d \mu & =\mu\left(\left\{i \in(0,1] / \alpha(i)=s_{r} \wedge X_{i}\left(\omega_{r}\right)=s_{v}\right\}\right)= \\
& =\mu\left(A_{r v}(\alpha, \omega)\right)=P_{r} P_{v}
\end{aligned}
$$

So

$$
P\left(\left\{\sigma \in \sum / \int_{(0,1]} \alpha_{r}(i) \alpha_{v}(\sigma(i)) d \mu=P_{r} P_{v}\right\}\right)=1
$$


so the random matching scheme satisfies also the Law of Large Numbers for types.

Example 1. Let us illustrate the previous theorem with an extremely simplified example. Assume there are only two different types of agents in the population, 1 and 2. Suppose that the assignment of types is particularly simple: $I_{1}(\alpha)=(0,0.5]$ and $I_{2}(\alpha)=(0.5,1]$. This way, the agents are already in half-open intervals. Otherwise, the first step in Theorem 4 is precisely to define measure-preserving bijections $\varphi_{r}^{1}$ between the sets $I_{r}(\alpha)$ and some half-open intervals.

We need the realizations of two independently uniformly distributed random variables taking values on $(0,1]$. Suppose, to illustrate the procedure, that these realizations are $\omega_{1}=0.5$ and $\omega_{2}=0.2$. For type 1 , this means the following steps are undertaken. First, expand $I_{1}(\alpha)$ to the interval $(0,1]$ through the application $x \rightarrow 2 x$. Second, add $\omega_{1}=0.5$ to all the elements of this interval, modulo 1 (i.e. subtract 1 if the result exceeds 1 ; this can be seen as "rolling a circumference"). Third, superpose the new set to the marked circumference, or rather to a half-open interval $(0,1]$ such that all numbers in $(0,0.5]$ are labeled " 1 " and the rest are labeled " 2 ". The original interval $(0,0.25]$ of agents of type 1 has been successively transformed in the sets $(0,0.5]$ and $(0.5,1]$ by this procedure, so they are now placed over "2" labels. This means they will be matched with agents of type 2 . The rest of the agents of type 1 will be matched with agents of type 1 . In the notation of the previous proof, this means $A_{11}(\alpha,(0.5,0.2))=(0.25,0.5]$ and $A_{12}(\alpha,(0.5,0.2))=(0,0.25]$.

Analogously, it is easy to compute the sets of agents of type 2 who will be matched with agents of type 1 or 2 , i.e. $A_{21}(\alpha,(0.5,0.2))=(0.5,0.65] \cup(0.9,1]$ and $A_{22}(\alpha,(0.5,0.2))=(0.65,0.9]$.

Now it is time to construct the actual matching. Agents in $A_{11}(\alpha,(0.5,0.2))$ have to be matched among themselves. Again, as this set is already an interval, the measure-preserving bijections used in the proof to transform it in an interval are straightforward, and the matching restricted to the set will simply prescribe $i \rightarrow$ $i+\frac{1}{2}(0.25) \forall i \in(0.25,0.375]$ and vice versa. Also, agents in $A_{22}(\alpha,(0.5,0.2))$ have to be matched among themselves according to the analogous mapping.

Agents in $A_{12}(\alpha,(0.5,0.2))$ have to be matched to agents in $A_{21}(\alpha,(0.5,0.2))$. Given that the form of these sets is quite simple, the actual measure-preserving bijections prescribed by the theorem are also simple, monotonically mapping $(0,0.25]$ into $(0.5,0.65] \cup(0.9,1]$. 
In summary, the actual matching will be:

$$
\sigma(i)= \begin{cases}i+0.5 & \text { if } i \in(0,0.15] \\ i+0.75 & \text { if } i \in(0.15,0.25] \\ i+0.125 & \text { if } i \in(0.25,0.375] \\ i-0.125 & \text { if } i \in(0.375,0.5] \\ i-0.5 & \text { if } i \in(0.5,0.65] \\ i+0.125 & \text { if } i \in(0.65,0.775] \\ i-0.125 & \text { if } i \in(0.775,0.9] \\ i-0.75 & \text { if } i \in(0.9,0.1]\end{cases}
$$

Note that the proportion of matchings between agents of type 1 is precisely $\frac{1}{2} \cdot \frac{1}{2}=$ $\frac{1}{4}$, between agents of type 2 is the same proportion, and between agents of different types is $2 \cdot \frac{1}{2} \cdot \frac{1}{2}=\frac{1}{2}$. This illustrates P3. Also, half the agents of type 1 are matched with agents of type 2, which illustrates $P 2$.

\section{Uniformity and ANONYmity Properties}

Both P4 and P2 can be seen as some sort of uniformity properties, formalizing the idea that all agents should face the same distribution over possible partners. We have already seen in the previous theorem that there exist random matching schemes satisfying $\mathrm{P} 2$, which is in fact the uniformity property actually used in many models. It seems that, analogously to $\mathrm{P} 5, \mathrm{P} 4$ is a too strong property.

Is easy to see, for instance, that the random matching scheme constructed in the previous theorem does not satisfy $\mathrm{P} 4$. Let $\varphi_{1}^{1}: I_{1}(\alpha) \rightarrow\left(0, P_{1}\right]$ to be the measurable and measure-preserving function mapping the set of agents of type $s_{1}$ into a half-open interval of the same measure, which is used in the proof of Theorem 4. Assume $0<P_{1}<\frac{1}{2}$ and consider the agent $i=\left(\varphi_{1}^{1}\right)^{-1}\left(\frac{1}{2} P_{1}\right)$. Define the set $E=\left(\varphi_{1}^{1}\right)^{-1}\left(\left(\frac{1}{2} P_{1}-P_{1}^{2}, \frac{1}{2} P_{1}+P_{1}^{2}\right]\right)$. According to Theorem 4 , the set of agents of type $s_{1}$ matched with agents of the same type, $A_{11}(\alpha, \omega)$ has measure $P_{r}^{2}$ (almost surely). According to $\mathrm{P} 2, \mu^{m}\left(\omega / i \in A_{11}(\alpha, \omega)\right)=P\left(\sigma(i) \in I_{1}(\alpha)\right)=P_{1}$. In this case, we would have $X_{i}(\omega)=s_{1}$ and, due to the correlation between the random variables $X_{i}$, it is easy to see that $A_{11}(\alpha, \omega) \subset E$ follows in this case (by definition of $E)$. Hence, $P(\sigma(i) \in E) \geq P\left(\sigma(i) \in I_{1}(\alpha)\right)=P_{1}$. But $\mu(E)=2 P_{1}^{2}<P_{1}$, a contradiction with $\mathrm{P} 4$.

Nevertheless, uniformity properties are not completely hopeless. It is not hard to prove that this difficulty arises only when considering agents and sets of agents of the same type, i.e. $i \in I_{r}(\alpha)$ and $E \subset I_{r}(\alpha)$. For the random matching scheme constructed in Theorem 4, the following, weaker property holds: 
(P4') (Restricted Proportional Law): $\forall i \in I_{r}(\alpha), \forall E \in \mathbb{B} / E \cap I_{r}(\alpha)=$ $\emptyset, P(\sigma(i) \in E)=\mu(E)$.

This is due to the fact that the randomization $\omega_{r}$ of agent $i$ and those of the agents in the set $E$ are then independent.

Another, more basic property would be to require the random matching scheme to preserve some sort of anonymity. This would mean that, given any agent $i$, and given two other agents $j, k$, the probability with which agent $i$ is matched with agent $j$ is equal to the probability with which agent $i$ is matched with agent $k$. Boylan (1992) shows that this property can never be satisfied in a countably infinite population, and thus it can be argued that no random matching scheme can be anonymous if the population is countably infinite. If the population is a continuum, though, this property can also be fulfilled, thanks to the idea of density. More concretely, the probability with which a given agent is matched with any other one can be assumed to be zero.

(P6) (Anonymity): $\forall i \in(0,1], \forall j \in(0,1] \backslash\{i\}, P(\sigma(i)=j)=0$.

If two agents $i, j$ are of different types $s_{r}, s_{v}$, this property holds in the construction of Theorem 4 because the random variables which determine the matching of $i$ and $j$ (i.e. $\omega_{r}$ and $\omega_{v}$ ) are independent. In the notation of the previous theorem, $i$ and $j$ can be thought of as independently uniformly distributed on $\left(0, P_{r} P_{v}\right]$, and thus, given $\varphi_{r v}$, the probability that $\varphi_{r v}(i)=j$ is zero.

But, taking again agents of the same type, the random matching scheme constructed in Theorem 4 does not satisfy P6. To see it, and relying again on the previous counterexample, recall that $\sigma_{\alpha}^{\omega}(i)=\left(\varphi_{11}^{-1} \circ \psi_{1} \circ \varphi_{11}\right)(i)$ if $i \in A_{11}(\alpha, \omega)$, with $\psi_{1}:\left(0, P_{1}^{2}\right] \rightarrow\left(0, P_{1}^{2}\right]$ such that $\psi_{1}(x)=x+\frac{1}{2} P_{1}^{2} \forall x \in\left(0, \frac{1}{2} P_{1}^{2}\right], \psi_{1}(x)=$ $x-\frac{1}{2} P_{1}^{2} \forall x \in\left(\frac{1}{2} P_{1}^{2}, P_{1}^{2}\right]$. If $\varphi_{11}$ is constructed through Lemma 3 , as $\varphi_{1}^{1}$ is, it is not difficult to see that, whenever $i \in A_{11}(\alpha, \omega)$, it will be matched either with agent $\left(\varphi_{1}^{1}\right)^{-1}\left(\varphi_{1}^{1}(i)+\frac{1}{2} P_{1}^{2}\right)$ or with agent $\left(\varphi_{1}^{1}\right)^{-1}\left(\varphi_{1}(i)-\frac{1}{2} P_{1}^{2}\right)$. So, these last two agents have strictly positive probability of being matched with agent $i$.

This problem is exclusively due to the arbitrary choice of $\frac{1}{2}$ as a focal point in the definition of $\psi_{1}$. To get rid of it, we can construct another random matching scheme along the following lines. Proceed as in Theorem 4, and add one new random variable uniformly distributed in the open interval $(0,1)$ and independent from $\omega$. Denote its realizations by $\omega_{m+1}$. When the matching restricted to the sets $A_{r r}(\alpha, \omega)$ have to be constructed, instead of using the arbitrary functions $\psi_{r}$, substitute them by

$$
\psi_{r}\left(\omega_{m+1}\right):\left(0, P_{r}^{2}\right] \rightarrow\left(0, P_{r}^{2}\right]
$$

such that $\psi_{r}\left(\omega_{m+1}\right)(x)=x+\omega_{m+1} P_{r}^{2}$ if this quantity is less than or equal to $P_{r}^{2}$, and $\psi_{r}\left(\omega_{m+1}\right)(x)=x+\left(\omega_{m+1}-1\right) P_{r}^{2}$ otherwise. This way, the focal point $\frac{1}{2}$ is 
substituted by one realization $\omega_{m+1}$ of a random variable independent of $\omega_{r}$, adding one further randomization which ensures $\mathrm{P} 6$ when $i, j$ are of the same type. In summary, we have proved the following result.

Proposition 5. For any assignment of types $\alpha$, there exists a random matching scheme satisfying $P 1, P 2, P 3$, and $P 6$.

This results shows an advantage of the continuum idealization over a countably infinite population.

\section{Repeated Matching Schemes}

Different models use random matching schemes for different purposes. Typically, the random matching occurs in the framework of a dynamical system, i.e., it takes place repeatedly. In general, it is postulated that a continuum of agents is randomly matched each period and types may change according to the realized matchings.

In order to apply Theorem 4 to a given model, it needs to be shown that if the assignment of types is given by a measurable function, as assumed, a random matching scheme is applied, and types change accordingly, then the new assignment of types will also be measurable (almost surely), so that Theorem 4 can be applied again.

The next corollary illustrates the point through a simple situation taken from Evolutionary Game Theory (see e.g. Boylan (1992) for details). Let an updating rule $\tau: S \times S \rightarrow S$ be a mapping describing the evolution of a random matching model. The interpretation of $\tau$ is as follows: If at time $t$ an individual of type $s_{r}$ is matched with an individual of type $s_{v}$, then at time $t+1$ the individual will be of type $\tau\left(s_{r}, s_{v}\right)$. In summary, $\tau$ contains the "story" under each specific model. Easy examples are derived from games like the well-known Rock-Scissors-Paper or the Hawk and Dove game.

Corollary 6. Let $\alpha$ be a (measurable) assignment of types, and let $\left(\sum, \mathcal{F}, P\right)$ be a random matching scheme as constructed in Theorem 4. Let $\alpha(\sigma, \tau)$ be the new assignment of types after application of a matching $\sigma$ and an updating rule $\tau$. Then, $P\left(\sigma \in \sum / \alpha(\sigma, \tau)\right.$ is measurable $)=1$, i.e., the new assignment is measurable ( $P$ almost surely).

Proof. Let $\alpha^{\prime}=\alpha(\sigma, \tau)$. In the notation of Theorem 4, let $\sigma=\sigma(\alpha, \omega) \in$ $\sum_{\alpha}$ and let $A_{r v}(\alpha, \omega)$ be the associated sets. Then, the new assignment of types is described by:

$$
\begin{aligned}
I_{r}\left(\alpha^{\prime}\right) & =\left\{i \in(0,1] / \tau(\alpha(i), \alpha(\sigma(j)))=s_{r}\right\}= \\
& =\cup\left\{A_{k v}(\alpha, \omega) / \tau\left(s_{k}, s_{v}\right)=s_{r}\right\}
\end{aligned}
$$


and these sets are obviously measurable because the sets $A_{k v}(\alpha, \omega)$ are measurable as shown in the proof of Theorem 4 , and the union is finite. So, $\alpha^{\prime}$ is measurable.

Example 2. Consider the following example, taken from Evolutionary Game Theory. Agents are of two types, 1 and 2. When an agent of type (strategy) $s_{r}$ plays against another agent of type $s_{v}$, he obtains a payoff $a_{r v}$. The payoff matrix $A=\left[a_{r v}\right]$ is:

$$
A=\left[\begin{array}{ll}
-1 & 2 \\
0 & 1
\end{array}\right]
$$

This is a version of the Hawk and Dove game (see e.g. [15, p. 27] or [10, p. 12]), which models the fight for a scarce resource.

A simple story (model), presented here only for expositional purposes, is as follows. Each period, agents are randomly matched in pairs to play the game. If two type 1 agents ("hawks") meet, they fight and obtain equal negative payoffs, so next period they both become type 2 agents ("doves"). If agents of different types meet, type 1 obtains the whole resource and thus both agents will become type 1 . If two type 2 agents meet, they share the resource and thus they will not change their type. An alternative, more biological story will interpret the payoffs in terms of offspring.

This story gives rise to an updating rule $\tau$ such that $\tau(1,1)=\tau(2,2)=2$ and $\tau(1,2)=\tau(2,1)=1$.

Suppose, for the sake of concreteness, that the initial assignment of types $\alpha$ and the first realization of the random matching scheme are as in Example1. There, we had that the set of agents of type 1 matched to agents of the same type was $A_{11}(\alpha, \omega)=(0.25,0.5]$. Thus, all these agents (or their offspring) will become type 2 according to $\tau$. Analogously, all agents in $A_{22}(\alpha, \omega)=(0.65,0.9]$ will remain type 2 , and all agents in $A_{12}(\alpha, \omega) \cup A_{21}(\alpha, \omega)=(0,0.25] \cup(0.5,0.65] \cup(0.9,1]$ will be of type 1 after application of the matching $\sigma$ and the updating rule $\tau$. In summary, the new assignment of types $\alpha^{\prime}$ will be:

$$
\alpha^{\prime}(i)= \begin{cases}1 & \text { if } i \in(0,0.25] \\ 2 & \text { if } i \in(0.25,0.5] \\ 1 & \text { if } i \in(0.5,0.65] \\ 2 & \text { if } i \in(0.65,0.9] \\ 1 & \text { if } i \in(0.9,1]\end{cases}
$$

which is obviously measurable. Observe that, in this case, the population frequencies of the two types have not changed. This is particular to this example and will be explained later. 


\section{Evolution of Population Frequencies}

A discrete time dynamical model will typically invoke random matching schemes an infinite number of times, which will involve constructing a probability space to describe the evolution of the population. Although, in principle, the above result guarantees the possibility of repeated random matching for any arbitrarily large but finite number of periods, one may run into problems when extending the scheme to an infinite number of periods.

In most of the applications, however, we are not interested in the actual assignment of types in the population as time goes by, but only in the evolution of the population frequencies. In fact, this is the classical formulation in Evolutionary Game Theory. Thus, the focus in this section will be to construct the probability spaces that allow us to trace the evolution of the population frequencies.

7.1. Rearranging the agents. Again, Lemma 3 may lead us to think that rearranging the agents from any given distribution of types into connected intervals is basically harmless. So, we could think in the following model: at each period, rearrange the agents in intervals (in the terms of the proof of Theorem 4, from $\alpha$ to $Z$, or, more intuitively, transform the population in the marked circumference), so that all the agents of a given type are in a connected, half-open interval of the appropriate measure. Then, apply to this new assignment of types the random matching scheme given by Theorem 4 .

Formally, given an assignment of types $\alpha$, define the "canonical" rearrangement $Z_{\alpha}$ by $Z_{\alpha}(i)=s_{r} \Leftrightarrow i \in\left(\sum_{j=1}^{r-1} \mu\left(I_{j}(\alpha)\right), \sum_{j=1}^{r} \mu\left(I_{j}(\alpha)\right)\right]$.

For the following result, recall the notation $\sigma(\alpha, \omega)=\sigma_{\alpha}^{\omega}$ for the matching obtained from an assignment of types $\alpha$ and a realization $\omega \in(0,1]^{m}$ using the procedure described in the proof of Theorem 4.

Lemma 7. Let $\Lambda=\left\{\alpha:(0,1] \rightarrow S / I_{r}(\alpha)\right.$ is measurable $\left.\forall s_{r} \in S\right\}$ be the set of all assignment of types, and let $B((0,1])$ be the set of all measure-preserving bijections from $(0,1]$ to itself. Then, there exists a mapping $\Psi: \Lambda \rightarrow B((0,1])$ such that, $\forall \alpha \in \Lambda, Z_{\alpha} \circ \Psi(\alpha)=\alpha$.

Proof. Given $\alpha \in \Lambda$, and for all $s_{r} \in S$, use lemma 3 to construct a measurepreserving bijection $\Psi_{r}(\alpha): I_{r}(\alpha) \rightarrow\left(\sum_{j=1}^{r-1} \mu\left(I_{j}(\alpha)\right), \sum_{j=1}^{r} \mu\left(I_{j}(\alpha)\right)\right]$. Then, define $\Psi(\alpha):(0,1] \rightarrow(0,1]$ by $\Psi(\alpha)(i)=\Psi_{r}(\alpha)(i)$ iff $i \in I_{r}(\alpha)$. Obviously, $\Psi(\alpha)$ is a measure-preserving bijection and, for all $i \in(0,1], Z_{\alpha}(\Psi(\alpha)(i))=\alpha(i)$.

Lemma 7 gives us a specific way to simultaneously rearrange all the agents of the population in such a way that all the agents of a given type are put together into a half-open interval of the same measure. Of course, this rearrangement is arbitrary, 
but, as it is done with measure-preserving bijections, it can be used back and forth. For example, given an assignment of types $\alpha$, the following two constructions are equivalent for all purposes: a) re-arrange the agents through $\Psi(\alpha)$ and use Theorem 4 to select a matching $\sigma\left(Z_{\alpha}, \omega\right)$; and b) use Theorem 4 to select a matching $\sigma\left(Z_{\alpha}, \omega\right)$, and apply to the population the matching $\sigma^{\prime}=\Psi(\alpha)^{-1} \circ \sigma\left(Z_{\alpha}, \omega\right) \circ \Psi(\alpha)$. That is, Lemma 7 can be applied to transform the matchings for $Z_{\alpha}$ into matchings for $\alpha$, so that we can work "as if" the population could be freely re-arranged.

An assignment of types of the form $Z_{\alpha}$ can be summarized by the vector of frequencies $f_{\alpha}=\left(\mu\left(I_{j}(\alpha)\right)\right)_{j=1}^{m}$, which is an element of the simplex $\Delta^{m}$. Conversely, given a vector of frequencies $f$, it is easy to define an assignment of types $Z_{f}$ by $Z_{f}(i)=s_{r} \Leftrightarrow i \in\left(\sum_{j=1}^{r-1} f_{j}, \sum_{j=1}^{r} f_{j}\right]$. For notational convenience, denote $F(\alpha)=f_{\alpha}$, and $Z(f)=Z_{f}$. Obviously, $Z_{\alpha}=Z(F(\alpha))$.

7.2. The trajectories. The trajectories of a system relying in infinitely repeated random matching will be, in principle, elements of the space $\left(S^{(0,1]} \times \sum\right)^{\mathbb{N}}$, that is, for each period $t$, the trajectory should specify both the assignment of types in the population and the matching according to which the agents are paired. In practice, and in view of the above discussion, it is better to consider trajectories as elements of the space $\left(\Delta^{m} \times(0,1]^{m}\right)^{\mathbb{N}}$, that is, for each period $t$, the trajectory would specify both the population frequencies as an element of the simplex $\Delta^{m}$ and the realization of $m$ i.i.d. uniform random variables on the interval $(0,1]$, which will uniquely determine the matching.

Thus, let $E=\Delta^{m} \times(0,1]^{m}$ be the state space of our process. Let again $\mathbb{B}^{m}$ be the Borel $\sigma$-algebra on $(0,1]^{m}$, and let $\mathbb{D}$ be the Borel $\sigma$-algebra on the simplex $\Delta^{m}$. Construct the product $\sigma$-algebra $\mathbb{E}=\mathbb{D} \otimes \mathbb{B}^{m}$.

7.3. Construction of the Probability Space. Given an updating rule $\tau: S \times$ $S \rightarrow S$, let $t: S^{(0,1]} \times \sum \rightarrow S^{(0,1]}$ be such that $\forall i \in(0,1], \forall \alpha \in S^{(0,1]}, \forall \sigma \in \sum$,

$$
t(\alpha, \sigma)(i)=\tau(\alpha(i), \alpha(\sigma(i)))
$$

Given an assignment of types $\alpha$ and a matching $\sigma$, this mapping uses the updating rule $\tau$ to obtain the new assignment of types, $t(\alpha, \sigma)$. That is, the mapping $t$ is the link which puts together a specific model summarized by an updating rule $\tau$ and a random matching specification to create a dynamical system.

In order to define a true probability space on trajectories, one needs to define a transition probability. We will do it using the "as if" approach described above, relying implicitly on Lemma 7 to work as if the population were constantly re-arranged in connected, half-open intervals on the basis of types. This allows us to keep track only of population frequencies. 
Let $Q: E \times \mathbb{E} \rightarrow[0,1]$ be such that for all $(f, \omega) \in E$ and all $(A, B) \in \mathbb{E}$,

$$
Q((f, \omega),(A, B))=\chi_{A}(F(t(Z(f), \sigma(Z(f), \omega)))) \mu^{m}(B)
$$

$Q\left((f, \omega),\left(f^{\prime}, \omega^{\prime}\right)\right)$ is the probability that if at time $t$ the population frequencies are $f$ and individuals are matched according to the matching obtained from $\omega$, then at time $t+1$ the population frequencies are $f^{\prime}$ and individuals are matched according to the matching obtained from $\omega^{\prime}$.

Lemma 8. The function $Q$ is a transition probability.

Proof. $Q$ will be a transition probability if for each $C \in \mathbb{E}, Q(\cdot, C)$ is measurable, and for each $(f, \omega) \in E, Q((f, \omega), \cdot)$ is a probability measure.

The second part is obvious, since both $\mu^{m}$ and $\chi_{(\cdot)}(F(t(Z(f), \sigma(Z(f), \omega))))$ are probability measures.

To see the first, fix $C=(A, B) \in \mathbb{E}$. Note that $\mu^{m}(B)$ is a constant, and that $\chi_{A}$ is a measurable function. Define $T: \Delta^{m} \times(0,1]^{m} \rightarrow \Delta^{m}$ by

$$
T(f, \omega)=F(t(Z(f), \sigma(Z(f), \omega))
$$

It would suffice to show that $T$ is measurable.

Let $D \in \mathbb{D}$. Then,

$$
T^{-1}(D)=\{(f, \omega) \in E / F(t(Z(f), \sigma(Z(f), \omega)) \in D\}
$$

But, by construction, given any $\omega$, the number of matches between agents of types $s_{r}$ and $s_{v}$ is always $f_{r} f_{v}$, so if we denote $m_{r}(f)=\sum\left\{f_{k} f_{v} / s_{k}, s_{v} \in S \wedge \tau\left(s_{k}, s_{v}\right)=s_{r}\right\}$, we have

$$
T^{-1}(D)=\left\{f \in \Delta^{m} / m(f) \in D\right\} \times(0,1]^{m}=m^{-1}(D) \times(0,1]^{m}
$$

Since $\tau$ is fixed, is obvious that the function $m: \Delta^{m} \rightarrow \Delta^{m}$ is measurable, so $T$ is also measurable and thus $Q(\cdot, C)$ is measurable.

Definition 3. A dynamical system on frequencies with random matching is a probability space $\left(\left(\Delta^{m} \times(0,1]^{m}\right)^{\mathbb{N}}, \bigotimes_{n \in \mathbb{N}} \mathbb{E}, P\right)$

Theorem 9. Given an initial condition $\left(f_{\mathbf{0}}, \omega_{\mathbf{0}}\right)$, there exists a dynamical system on frequencies with random matching such that, at each period t, the random matching scheme satisfies P1, P2 and P3 (almost surely). In particular, the number of matchings between individuals of types $s_{r}$ and $s_{v}$ is $f_{r}(t) f_{v}(t) \forall s_{r}, s_{v} \in S$ (almost surely), and thus the system can be described by the (deterministic) system of equations

$$
f_{r}(t+1)=\sum\left\{f_{k}(t) f_{v}(t) / s_{k}, s_{v} \in S \wedge \tau\left(s_{k}, s_{v}\right)=s_{r}\right\}
$$


Proof. In view of Lemma 8, the probability space on trajectories can be constructed applying the Ionescu-Tulcea theorem (see e.g. [12, Proposition V.1.1] or [1, Theorem 2.7.2]), which states that there is a unique probability $Q^{*}$ over $\left(E^{\mathbb{N}}, \bigotimes_{n \in \mathbb{N}} \mathbb{E}\right)$ such that for every finite dimensional rectangle $\prod_{j=1}^{J} C_{j} \times \prod_{n=J+1}^{\infty} E$,

$$
\begin{gathered}
Q^{*}\left(\prod_{j=1}^{J} C_{j} \times \prod_{n=J+1}^{\infty} E\right)= \\
\int_{C_{1}} Q\left(\left(f_{0}, \omega_{0}\right),\left(f_{1}, \omega_{1}\right) ; d\left(f_{1}, \omega_{1}\right)\right) \ldots \int_{C_{J}} Q\left(\left(f_{J-1}, \omega_{J-1}\right),\left(f_{J}, \omega_{J}\right) ; d\left(f_{J}, \omega_{J}\right)\right)
\end{gathered}
$$

The rest follows from the above discussion.

For any measurable $\alpha:(0,1] \rightarrow S$, Lemma 7 allows us to specify a measurepreserving bijection $\Psi(\alpha):(0,1] \rightarrow(0,1]$ such that, for all $s_{r} \in S, \Psi\left(I_{r}(\alpha)\right)=$ $\left(\sum_{j=1}^{r-1} \mu\left(I_{j}(\alpha)\right), \sum_{j=1}^{r} \mu\left(I_{j}(\alpha)\right)\right]$. Taking this specification of measure-preserving bijections, it is possible to transform back the trajectories of the above system into elements of $\left(S^{(0,1]} \times \sum\right)^{\mathbb{N}}$.

Start with a given assignment of types $\alpha_{0}$. Take $f_{0}=F\left(\alpha_{0}\right)$ and any $\omega_{0} \in(0,1]^{m}$ as initial condition for the above result. Given a trajectory of the above system, that is, a realization $(f(t), \omega(t))_{t=0}^{\infty} \in\left(\Delta^{m} \times(0,1]^{m}\right)^{\mathbb{N}}$, it is possible to uniquely obtain a trajectory as an element $(\alpha(t), \sigma(t))_{t=0}^{\infty} \in\left(S^{(0,1]} \times \sum\right)^{\mathbb{N}}$ as follows:

$$
\begin{aligned}
\alpha(0)= & \alpha_{0} ; \sigma(0)=\Psi\left(\alpha_{0}\right)^{-1} \circ \sigma\left(Z\left(f_{0}\right), \omega_{0}\right) \circ \Psi\left(\alpha_{0}\right) \\
\alpha(1)= & t\left(\alpha_{0}, \sigma(0)\right) ; \sigma(1)=\Psi(\alpha(1))^{-1} \circ \sigma(Z(f(1)), \omega(1)) \circ \Psi(\alpha(1)) \\
& \ldots \\
\alpha(t+1)= & t(\alpha(t), \sigma(t)) ; \sigma(t+1)=\Psi(\alpha(t))^{-1} \circ \sigma\left(Z(f(t)), \omega_{1}\right) \circ \Psi(\alpha(1))
\end{aligned}
$$

Moreover, it is clear that for all $t, f(t)=F(\alpha(t))$.

Call $\Gamma$ to the mapping just described. It is clear that any $(\alpha(t), \sigma(t))_{t=0}^{\infty} \in$ $\Gamma\left(\left(\Delta^{m} \times(0,1]^{m}\right)^{\mathbb{N}}\right)$ uniquely determines a trajectory in $\left(\Delta^{m} \times(0,1]^{m}\right)^{\mathbb{N}}$, so $\Gamma$ is injective. In summary,

Corollary 10. Given an initial condition, there exists a probability space defined on $\left(S^{(0,1]} \times \sum\right)^{\mathbb{N}}$ satisfying the properties enumerated in Theorem 9 .

Proof. Apply Theorem 9 and take the $\sigma$-algebra generated by $\Gamma^{-1}\left(\bigotimes_{n \in \mathbb{N}} \mathbb{E}\right)$ and the probability measure $P \circ \Gamma$.

This corollary gives an actual construction of a probability space which can be used to formalize a wide range of models in such a way that: 
1. At each moment in time, the population is matched according to a true random matching scheme, that is, there is uncertainty on the individual level.

2. The actual evolution of the population gives rise to a stochastic system.

3. The evolution of the population frequencies that this system induces can be described through a system of deterministic equations, that is, there is no uncertainty on the aggregate level.

Of course, property P6 could also be guaranteed basing the construction on Proposition 5 instead of on Theorem 4 .

Example 3. Let us continue the thread of Examples 1 and 2. If individuals of two different types, of frequencies $f_{1}(t)$ and $f_{2}(t)$ are repeatedly randomly matched and types change according to the updating rule postulated in Example 2, then Theorem 9 states that the evolution of $f_{1}(t)$ and $f_{2}(t)$ can be described through the following deterministic system:

$$
\begin{aligned}
f_{1}(t+1) & =\sum\left\{f_{k}(t) f_{v}(t) / s_{k}, s_{v} \in S \wedge \tau\left(s_{k}, s_{v}\right)=1\right\}= \\
& =f_{1}(t) f_{2}(t)+f_{2}(t) f_{1}(t)=2 f_{1}(t) f_{2}(t) \\
f_{2}(t+1) & =\ldots=f_{1}(t)^{2}+f_{2}(t)^{2}
\end{aligned}
$$

Note that $\left(\frac{1}{2}, \frac{1}{2}\right)$ is a fixed point of this system, which explains why in the previous examples, starting with these population frequencies we obtain the same again.

Example 4. Consider the Rock-Scissors-Paper symmetric game. There are three types of agents (strategies), $R, S$, and $P$, and the payoff matrix is:

$$
\begin{array}{llll} 
& R & S & P \\
R & 1 & 2 & 0 \\
S & 0 & 1 & 2 \\
P & 2 & 0 & 1
\end{array}
$$

with the interpretation that $a_{r v}$ is the number of offspring of type $s_{r}$ that an agent of this type produces when matched with another agent of type $s_{v}$. This story (which can be found in Boylan (1992)) is captured by an updating rule such that $\tau\left(s_{r}, s_{v}\right)=s_{r}$ if $a_{r v} \neq 0$ and $\tau\left(s_{r}, s_{v}\right)=s_{v}$ otherwise. Theorem 9 states that, when a continuum of agents is repeatedly randomly matched to play this game and types are updated according to this model, then the evolution of the population frequencies $f_{R}, f_{S}, f_{P}$ is described (almost surely) by the equations

$$
\begin{aligned}
& f_{R}(t+1)=f_{R}(t)^{2}+2 f_{R}(t) f_{S}(t) \\
& f_{S}(t+1)=f_{S}(t)^{2}+2 f_{S}(t) f_{P}(t) \\
& f_{P}(t+1)=f_{P}(t)^{2}+2 f_{P}(t) f_{R}(t)
\end{aligned}
$$


which, in this case, coincide with the well-known Replicator Dynamics (in discrete time) for the above payoff matrix.

\section{Discussion}

Many models of random matching postulate a continuum of agents and informally invoke a Law of Large Numbers to justify that:

1. The probability with which a given agent is matched with agents of a given type equals the proportion of agents of that type in the population.

2. The proportion of matches between agents of two given types is equal to the "expected number" of such matches when all matches are equally likely.

Nevertheless, the invoked Law of Large Numbers may not hold, and, in fact, it could be hard just to formally state the above assumptions.

This paper provides a foundation for such models, constructing random matching schemes which verify the desired properties. However, it is also shown that this is possible only if the random matching schemes depend on the assignment of types.

It should be noted that the random matching schemes constructed in Theorem 4 do not satisfy "full independence" of the types of the partners for two given individuals of the same type. However, one could easily argue that this is irrelevant, because the very concept of matching destroys the most basic independence aspirations. After all, if agent $i$ is matched with agent $j$, then agent $j$ has to be matched with agent $i$.

When the Law of Large Numbers is invoked, the purpose is to approximate the very complex stochastic system to which random matching gives rise by a deterministic system. Theorem 9 does precisely that, focusing on the evolution of population frequencies, and thus creates the link between the random matching "story" of certain dynamical systems and their deterministic formulation.

This paper should not be viewed as a justification for the informal use of a Law of Large Numbers in random matching of a continuum of agents, but rather as a warning call. In this field, "intuitively obvious" assumptions may be false. And, even if these assumptions can be established for specific constructions, it could take a fair amount of work to actually construct the processes that are usually left unspecified.

In fact, there are two possible different interpretations of the results in this paper.

The first interpretation focuses on the impossibility results, and very specially on the impossibility to construct random matching schemes independently of the assignment of types. In finite population models, random matching works in a straightforward way. The fact that in infinite population models it is necessary to actually 
design the random matching scheme, and that the properties of the system may depend on the specific scheme, may lead us to conclude that infinite population models (whether countable or not) are not the appropriate way to think about large populations, because they cannot be the "limit" of finite population models. Of course, this interpretation favors the analysis of large population models through limit theorems. Nevertheless, it is arguable whether the difficulties disappear in this approach or are merely obscured by the fact that the "limit model" may not exist.

The second interpretation focuses on the possibility results: the models which use random matching of a continuum of agents are possible, and thus it is technically justified to work with them. So, if the continuum is considered as a convenient idealization of the idea of a large population, there can be no objections to the use of random matching, and the underlying technical problems will not obscure the conceptual analysis.

\section{EXTENSIONS}

Random matching models are quite frequent in the economic literature. Thus, many of them depart from the basic framework considered here. Nevertheless, it should be clear that the random matching schemes constructed in this work can be used as building blocks for more complex models. Let us enumerate some extensions.

1. Multipopulation models. Sometimes, agents from different populations are randomly matched, e.g. to play asymmetric games (see Weibull (1995), Chapter 5 ) or in buyers-sellers models (see Peters $(1991,1997)$ ). Actually, this case is easier than the one-population framework. For instance, in a two-populations model the random matching scheme will work as follows: apply the "roulette method" to each population, but then place the resulting sets over the "marked circumference" corresponding to the other population. Then, we have the corresponding sets $A_{r v}(\alpha, \beta)$ where $\alpha, \beta$ are the assignments of types in populations 1 and 2 respectively, $s_{r}$ is any type in population 1 , and $s_{v}$ is any type in population 2. The situation is now easier because there are no sets $A_{r r}(\alpha, \beta)$ having to be matched among themselves. In fact, this will allow us to show that property $\mathrm{P} 4$ also holds without further modifications.

2. Stochastic updating rules. A more general model would allow for stochastic laws of motion, capturing e.g. statements about the probability that an agent becomes (or produces offspring) of a certain type, given the actual outcome of the game he has played. A similar interpretation underlies the Replicator Dynamics: "individuals reproduce their kind asexually, in numbers proportional to their fitnesses" (Maynard Smith (1982), p. 12). This could be reflected by a 
stochastic updating rule, i.e. a measurable function $\tau: S \times S \times(0,1] \rightarrow S$. Of course, if we require that each agent has an i.i.d. random variable, we will face again the problem of the inexistence of a Law of Large Numbers for a continuum of random variables. But it is possible to capture these ideas incorporating into the random matching scheme specific splittings of the sets $A_{r v}(\alpha, \omega)$ reflecting the appropriate proportions. E.g., if the model calls for a probability $\frac{1}{2}$ of becoming type 2 when an agent of type 1 meets another agent of type 2 , then the random matching scheme would have to specify a measurable splitting of the set $A_{12}(\alpha, \omega)$ in two subsets of the same measure.

3. Idiosyncratic environment. Harrington (1997) describes a model where each pair of randomly matched individuals observe a different realization of a random variable representing a changing environment. Of course, this again has the problem of generating a continuum of random variables to which no Law of Large Numbers applies. Again, this problem could be avoided if the random matching scheme specifies the appropriate splittings of the sets $A_{r v}(\alpha, \omega)$.

\section{Appendix One: Proof of Lemma 3}

Lemma 5. Let $E_{1}, E_{2} \in \mathbb{B} / \mu\left(E_{1}\right)=\mu\left(E_{2}\right)>0$. Then, $\exists \varphi: E_{1} \rightarrow E_{2}$ bijective and measurable with measurable inverse and such that $\forall A \in \mathbb{B} \cap E_{2}, \mu\left(\varphi^{-1}(A)\right)=$ $\mu(A) . \varphi$ is called a measure-preserving bijection.

Proof. It suffices to show that, given any measurable set $E \subset(0,1]$ with measure $\mu(E)=P$, there exists a measure-preserving bijection $\varphi: E \rightarrow(0, P]$.

Start with the trace measure space $\left(E, \mathbb{B}_{E}, \mu_{E}\right)$ as a subspace of $((0,1], \mathbb{B}, \mu)$.

First, note that, as the cardinality of $E$ is that of the continuum, there exists a Borel isomorphism $f: E \rightarrow(0, P]$, i.e., $f$ is a bijective measurable function with measurable inverse $f^{-1}$ (See e.g. $[4$, Theorem 8.6.3]). This defines the measure space $\left((0, P], \mathbb{B}_{(0, P]}, \nu\right)$, where $\nu=\mu_{E} \circ f^{-1}$. Note that this measure has no atoms.

Now, define the mapping $g:\left((0, P], \mathbb{B}_{(0, P]}, \mu_{(0, P]}\right) \rightarrow\left((0, P], \mathbb{B}_{(0, P]}, \nu\right)$ by

$$
g(x)=\inf \{t \in(0, P] / \nu((0, t]) \geq x\}
$$

We turn now to the analysis of the properties of $g$.

1. $\nu((0, g(x)])=x \forall x \in(0, P]$.

Let $g(x)=t>0$. If $x<\nu((0, t])$, let $n^{*} \in \mathbb{N} / t-\frac{1}{n}>0$. Then,

$$
x<\nu((0, t])=\nu((0, t))=\nu\left(\cup_{n=n^{*}}^{\infty}\left(0, t-\frac{1}{n}\right]\right)=\lim _{n \rightarrow \infty} \nu\left(\left(0, t-\frac{1}{n}\right]\right) \leq x
$$


where the first equality comes from the fact that $\nu$ is nonatomic, the second is obvious, the third is implied by the fact that $\left\{\left(0, t-\frac{1}{n}\right]\right\}$ is an increasing: sequence of measurable sets, and the last inequality is implied by the definition of infimum. This yields a contradiction which proves the claim.

If $g(x)=0$, then $\nu\left(\left(0, \frac{1}{n}\right]\right) \geq x \forall n \in \mathbb{N}$ and thus $x=0$, a contradiction.

2. $g$ is bijective.

First, to see that $g$ is injective, let $x_{1}, x_{2} \in(0, P]$. If $g\left(x_{1}\right)=g\left(x_{2}\right)$, then $x_{1}=\nu\left(\left(0, g\left(x_{1}\right)\right]\right)=\nu\left(\left(0, g\left(x_{2}\right)\right]\right)=x_{2}$

It remains to show that $g$ is surjective: let $t \in(0, P]$. We need to show that $\exists x \in(0, P] / g(x)=t$, i.e., $\nu((0, t])=x$.

Let, thus, $x=\nu((0, t])$. Then, $x=\nu((0, g(x)])=\nu((0, t]) \Rightarrow g(x)=t$

So, $g$ is bijective and $g^{-1}=h$ defined by $h(t)=\nu((0, t])$.

3. $g$ is non-decreasing.

Let $x_{1}, x_{2} \in(0, P] / x_{1}<x_{2}$, and suppose that $g\left(x_{1}\right) \geq g\left(x_{2}\right)$. Then, $\left(0, g\left(x_{2}\right)\right] \subset$ $\left(0, g\left(x_{1}\right)\right] \Rightarrow x_{2}=\nu\left(\left(0, g\left(x_{2}\right)\right]\right) \leq \nu\left(\left(0, g\left(x_{1}\right)\right]\right)=x_{1}<x_{2}$, a contradiction.

So $g$ is non-decreasing and hence measurable.

4. The mapping $h=g^{-1}$ is continuous.

Let $t \in(0, P)$ :

- Let $\left\{t_{n}\right\} \rightarrow t / t_{n}<t \forall n$. Then,

$$
\begin{aligned}
\lim _{n \rightarrow \infty} h\left(t_{n}\right) & =\lim _{n \rightarrow \infty} \mu_{E}\left(f^{-1}\left(\left(0, t_{n}\right]\right)\right)=\mu_{E}\left(\cup_{n=1}^{\infty} f^{-1}\left(\left(0, t_{n}\right]\right)\right)= \\
& =\mu_{E}\left(f^{-1}\left(\cup_{n=1}^{\infty}\left(0, t_{n}\right]\right)\right)=\mu_{E}\left(f^{-1}((0, t))\right)= \\
& =\mu_{E}\left(f^{-1}((0, t])\right)=h(t)
\end{aligned}
$$

- Let $\left\{t_{n}\right\} \rightarrow t / t_{n}>t \forall n$. Then,

$$
\begin{aligned}
\lim _{n \rightarrow \infty} h\left(t_{n}\right) & =\lim _{n \rightarrow \infty} \mu_{E}\left(f^{-1}\left(\left(0, t_{n}\right]\right)\right)=\mu_{E}\left(\cap_{n=1}^{\infty} f^{-1}\left(\left(0, t_{n}\right]\right)\right)= \\
& =\mu_{E}\left(f^{-1}\left(\cap_{n=1}^{\infty}\left(0, t_{n}\right]\right)\right)=\mu_{E}\left(f^{-1}((0, t])\right)=h(t)
\end{aligned}
$$

where the third equality relies on the fact that $f$ is bijective.

So $g^{-1}$ is continuous and hence measurable. ${ }^{6}$

\footnotetext{
${ }^{6}$ This fact could have been obtained directly applying [4, Proposition 8.3.5], which states that a bijective and measurable mapping between measurable subsets of Polish spaces has a measurable inverse.
} 
Define now $\varphi=g^{-1} \circ f:\left(E, \mathbb{B}_{E}, \mu_{E}\right) \rightarrow\left((0, P], \mathbb{B}_{(0, P]}, \mu_{(0, P]}\right)$

It remains to show that $\forall A \in \mathbb{B}_{(0, P]}, \mu_{E}\left(\varphi^{-1}(A)\right)=\mu_{(0, P]}(A)$. As $\mathbb{B}_{(0, P]}$ is a Borel $\sigma$-algebra, it is enough to establish the property for the intervals $(0, b]$.

$$
\mu_{E}\left(\varphi^{-1}((0, b])\right)=\mu_{E}\left(f^{-1}(g((0, b]))\right)=\nu(g((0, b]))
$$

But, given $t \in(0, b]$, as $g$ is non-decreasing, $g(t) \leq g(b)$. Moreover, as $g$ is bijective, $g((0, b])=(0, g(b)]$. So,

$$
\nu(g((0, b]))=\nu((0, g(b)])=b=\mu_{(0, P]}((0, b])
$$

So $\varphi$ is a measure-preserving bijection.

\section{Appendix Two: The Law of Large Numbers}

McLennan and Sonnenschein (1991) state the tentative Law of Large Numbers for random matching of a continuum of agents as follows:

(P5) $\mu\left(E_{1} \cap \sigma\left(E_{2}\right)\right)=\mu\left(E_{1}\right) \mu\left(E_{2}\right) \forall E_{1}, E_{2} \in \mathbb{B} P$-almost surely.

This is precisely the approach taken here. But, clearly, this property is too strong, since Proposition 1 shows that, under P1, no matching can satisfy it.

A different, apparently weaker statement would be the following:

(P5') $\forall E_{1}, E_{2} \in \mathbb{B}, P\left(\sigma \in \sum / \mu\left(E_{1} \cap \sigma\left(E_{2}\right)\right)=\mu\left(E_{1}\right) \mu\left(E_{2}\right)\right)=1$.

$\mathrm{P} 5$ requires that there is a set of matching of measure one such that, for all Borel subsets $E_{1}, E_{2}$, the measure of the agents in $E_{1}$ who are matched with agents in $E_{2}$ is the product of the measures of the two sets. ( $\left.5^{\prime}\right)$ only requires, that, given two Borel sets, the probability with which this property holds for them is one. It could be argued that, since P5 is hopeless, P5' should be understood as the appropriate formulation of a Law of Large Numbers in this framework.

Nevertheless, notice that the important result here is the fact random matching schemes have to depend on the assignment of types (Corollary 2). This result remains untouched, because the above discussion does not affect P3: notice that writing down P5 and P5' to hold only for the sets of agents of the same type give precisely the same property, since the number of sets involved is finite. Thus, if P5' was our choice, it will still be impossible to fulfill keeping independence from the assignment of types.

But, in fact, the situation is even more clear because, despite its apparent weakness, P5' implies P5: given P5', it is immediate to see that there exists a set of matchings of measure one simultaneously verifying the required property for all pairs of intervals of the form $(a, b]$ with $a, b$ rational numbers (this is because there is a countable number of such intervals). But, then, it is easy to conclude that this set of matchings verifies the required property for any arbitrary pair of Borel sets. 


\section{REFERENCES}

[1] Ash, R.B., "Real Analysis and Probability," Academic Press, New York, 1972.

[2] Boylan, R.T., Laws of Large Numbers for Dynamical Systems with Randomly Matched Individuals, Journal of Economic Theory 57 (1992), 473-504.

[3] Boylan, R.T., "Continuous Approximations of Dynamical Systems with Randomly Matched Individuals," Journal of Economic Theory 66 (1995), 615-625.

[4] Cohn, D.L., "Measure Theory," Birkhäuser, Boston, 1980.

[5] Feldman, M., \& C. Gilles, "An Fxpository Note on Individual Risk without Aggregate Uncertainty," Journal of Economic Theory 35 (1985), 26-32.

[6] Gilboa, I., \& A. Matsui, "A Model of Random Matching," Journal of Mathematical Economics 21 (1992), 185-197.

[7] Harrington, J.E. Jr., "The Social Selection of Flexible and Rigid Agents," American Economic Review, forthcoming.

[8] Judd, K.L., "The Law of Large Numbers with a Continuum of IID Random Variables," Journal of Economic Theory 35 (1985), 19-25.

[9] Matsui, A., \& K. Matsuyama, "An Approach to Equilibrium Selection," Journal of Economic Theory 65 (1995), 415-434.

[10] Maynard Smith, J., "Evolution and the Theory of Games," Cambridge University Press, Cambridge, 1982.

[11] McLennan, A., \& H. Sonnenschein, "Sequential Bargaining as a Noncooperative Foundation for Walrasian Equilibrium," Econometrica 59 (1991), 1395-1424.

[12] Neveu, J., "Bases Mathématiques du Calcul des Probabilités," 2nd edition, Masson, Paris, 1980.

[13] Peters, M., "Fx Ante Price Offers in Matching Games Non-Steady States," Econometrica 59 (1991), 1425-1454.

[14] Peters, M., "On the Equivalence of Walrasian and Non-Walrasian Equilibria in Contract Markets: the Case of Complete Contracts," Review of Economic Studies 64 (1997), 241-264.

[15] Weibull, J.W.,"Evolutionary Game Theory", MIT Press, Cambridge, Mass., 1995 . 